\title{
Abdominopelvic Metastasis of Endometrial Serous Carcinoma Initially Misdiagnosed as Early-Stage Low-Grade Endometrioid Carcinoma: The Importance of Recognizing Minimal Uterine Serous Carcinoma
}

\author{
Jiheun Han Hyun-Soo Kim \\ Department of Pathology and Translational Genomics, Samsung Medical Center, \\ Sungkyunkwan University School of Medicine, Seoul, South Korea
}

\section{Keywords}

Endometrium $\cdot$ Minimal uterine serous carcinoma $\cdot$ Endometrioid carcinoma $\cdot$ Metastasis

\begin{abstract}
Minimal uterine serous carcinomas (MUSCs) include serous carcinomas with invasion confined to the endometrium (superficial serous carcinoma) and those without stromal invasion (serous endometrial intraepithelial carcinoma). Although these tumors are confined to the endometrium proper, they have highly metastatic potential for disseminating to extra-uterine sites. We report here a case of MUSC that was initially misdiagnosed as early-stage low-grade endometrioid carcinoma but later metastasized to the abdominopelvic peritoneum. The patient was a 61-year-old woman who was diagnosed with grade 1 endometrioid carcinoma of the endometrium and underwent total hysterectomy. Because the tumor was confined to the endometrium (International Federation of Gynecology and Obstetrics stage IA), no further treatment was performed. However, several metastatic tumor masses were detected in the vaginal stump and abdominopelvic peritoneum 7 years after the surgery. Histologically, the metastatic tumor tissues showed high-grade carcinoma. A review of previous hysterectomy slides showed multiple separate foci of atypical glandular proliferation measuring up to $0.8 \mathrm{~cm}$ in the greatest dimension and consisting of markedly atypical cells involving the surface and atrophic glands. The tumor showed a predominantly glandular architecture without evident papillary growth or stromal invasion. However, it had large, pleomorphic nuclei showing a high nuclear-to-cytoplasmic ratio, conspicuous eosinophilic nucleoli, and numerous mitotic figures. Characteristically, the tumor showed marked nuclear atypia immediately appreciated at low magnification in the background of well-formed glandular structures, indicating a significant discordance between nuclear and architectural features. On immunostaining, both
\end{abstract}


the uterine and metastatic tumor tissues exhibited diffuse and strong p16 expression and mutant pattern of p53 expression, confirming the diagnosis of serous carcinoma. In summary, the case findings support that failure to preoperatively recognize high-risk endometrial carcinoma is associated with worse outcomes. Complete surgical staging and accurate pathological diagnosis are critical for patients with serous carcinoma even at the early clinical stage.

(C) 2020 The Author(s).

Published by S. Karger AG, Basel

\section{Introduction}

Uterine serous carcinoma is the prototypic type II endometrial carcinoma. It is a clinically aggressive tumor that typically occurs in the atrophic endometrium of postmenopausal women. Uterine serous carcinoma often presents with extra-uterine spread and has a high risk for recurrence and metastasis [1]. In contrast to the more common endometrioid carcinoma, serous carcinoma behaves aggressively even at the absence of other risk factors for poor prognosis in endometrial carcinoma.

A non-invasive immediate precursor of uterine serous carcinoma, that is, serous endometrial intraepithelial carcinoma, has been recently identified [2, 3]. Superficial serous carcinoma is defined as a serous carcinoma with early stromal invasion that is focal and confined to an endometrial polyp or endometrium proper. Because these tumors share similar clinical behaviors and histological features, and distinction between them based on the identification of stromal invasion is difficult. Serous endometrial intraepithelial carcinoma and superficial serous carcinoma of $<1 \mathrm{~cm}$ are collectively classified as minimal uterine serous carcinoma (MUSC) [4]. Despite the non-invasive or limited presence of invasive carcinoma in MUSC, it has been reported to be frequently associated with concurrent extra-uterine metastases.

We describe here a rare case of MUSC that was initially misdiagnosed as early-stage lowgrade endometrioid carcinoma. The patient developed multifocal abdominopelvic peritoneal metastases 7 years after surgery, and a retrospective review of hysterectomy slides revealed that the uterine tumor was MUSC, not an endometrioid carcinoma. Such a histological misinterpretation and a discrepancy between initial pathological stage and clinical outcome deserves greater attention both in clinical practice and in exploring the pathogenesis of this highly malignant disease.

\section{Case Presentation}

A 61-year-old postmenopausal woman presented with an abnormal sonographic appearance detected during routine pelvic ultrasonography. She had no history of vaginal bleeding or abdominal distention. Her previous medical or gynecological history was also unremarkable. Transvaginal ultrasonography revealed an irregular endometrial thickening (up to $12.5 \mathrm{~mm}$ ) with cystic change. She was accordingly diagnosed with adenocarcinoma based on the endometrial curettage specimen. However, magnetic resonance imaging revealed no visible lesion in the endometrial cavity, lymph nodes, and extra-uterine sites. The preoperative serum cancer antigen (CA) 125 level was also within the normal limit (3.9 U/ $\mathrm{mL}$ ). The patient underwent total laparoscopic hysterectomy with bilateral salpingo-oophorectomy and bilateral pelvic lymph node dissection.

Grossly, the uterus was small and atrophic, measuring $7.9 \times 5.5 \times 3 \mathrm{~cm}$ and weighing 69 g. An endometrial polyp $(2.5 \times 1.3 \mathrm{~cm})$ and an intramural leiomyoma $(0.6 \times 0.4 \mathrm{~cm})$ were identified. The non-polypoid endometrium and cervix had no remarkable lesion. The bilateral

\section{Karger'}



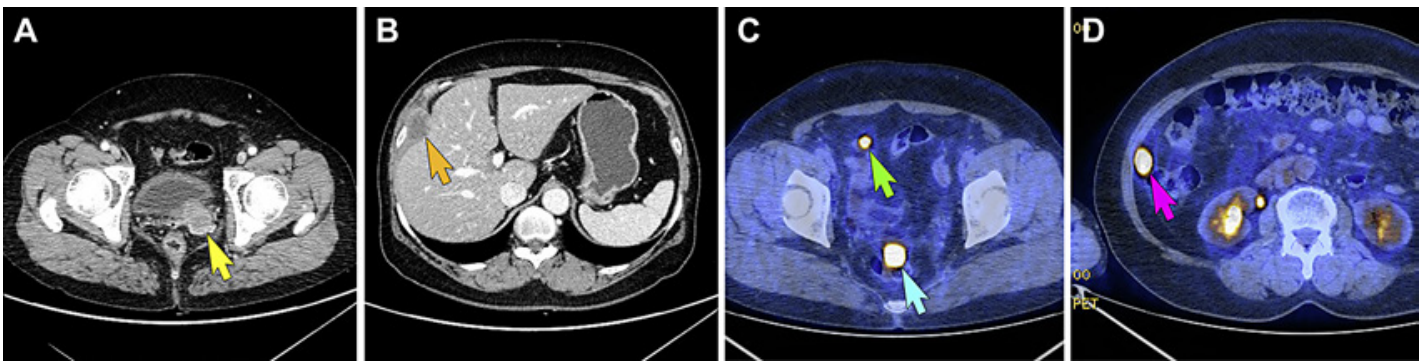

Fig. 1. Imaging findings. Computed tomography and positron emission tomography-computed tomography revealed metastatic tumor masses in the vaginal stump (A), right perihepatic space (B), pelvic peritoneum (C), and right paracolic gutter (D).

ovaries and salpinges were also unremarkable. Based on the presence of well-differentiated adenocarcinoma that was confined within the endometrium proper, the histological diagnosis of grade 1 endometrioid carcinoma was established. There was no evidence of myometrial or lymphovascular invasion. The bilateral pelvic lymph nodes, ovaries, and salpinges were free of tumor. Given that the International Federation of Gynecology and Obstetrics was stage IA, the patient did not receive postoperative adjuvant therapy and was only indicated for surveillance with annual outpatient follow-up.

However, 7 years after the surgery, a $2.7-\mathrm{cm}$ mass was detected in the left vaginal stump. Computed tomography revealed several metastatic tumor masses in the abdominopelvic peritoneum (Fig. 1A, B). Positron emission tomography-computed tomography revealed hypermetabolic lesions in the vaginal stump, right perihepatic space, right paracolic gutter, and pelvic peritoneum (Fig. 1C, D). The serum CA 125 level was also elevated up to $101.8 \mathrm{U} /$ $\mathrm{mL}$. At the last follow-up at 4.5 years postoperatively, there was no evidence of recurrent disease on imaging and the serum CA 125 level was within normal range. A punch biopsy was performed for the vaginal stump mass. Representative photomicrographs showing histological features and immunophenotype of the vaginal lesions are shown in Figure 2. Histologically, the tumor consisted of solid and papillary structures lined by markedly atypical cells with marked nuclear enlargement and pleomorphism and numerous mitotic figures. Immunohistochemically, the tumor cells displayed diffuse and strong nuclear immunoreactivities for p16 and p53, compatible with metastatic serous carcinoma of endometrial origin. The expressions for estrogen and progesterone receptors were focal and weak.

We reviewed previous slides obtained from the hysterectomy specimen. There were multiple separate foci of atypical glandular proliferation measuring up to $0.8 \mathrm{~cm}$ in the greatest dimension and consisting of markedly atypical cells involving the surface and the lining of atrophic endometrial glands located adjacent to the endometrial polyp. Representative photomicrographs showing histological features and immunophenotype of the vaginal stump mass are shown in Figure 3. The transition between the tumor and non-neoplastic endometrium was abrupt, similar to that seen in adenocarcinoma in situ of the endocervix. The tumor displayed predominantly well-formed glandular architecture without evident papillary growth or stromal invasion. However, the individual tumor cells possessed large, pleomorphic nuclei showing a high nuclear-to-cytoplasmic ratio, conspicuous eosinophilic nucleoli, and numerous mitotic figures. Characteristically, the tumor showed marked nuclear atypia readily appreciated at low magnification in the background of well-differentiated glandular structures, indicating a significant discordance between nuclear and architectural features. The tumor cell nuclei showed diffuse and strong p 16 immunoreactivity and missense mutation pattern of p53 expression, compatible with serous carcinoma. 


\section{Case Reports in Oncology}

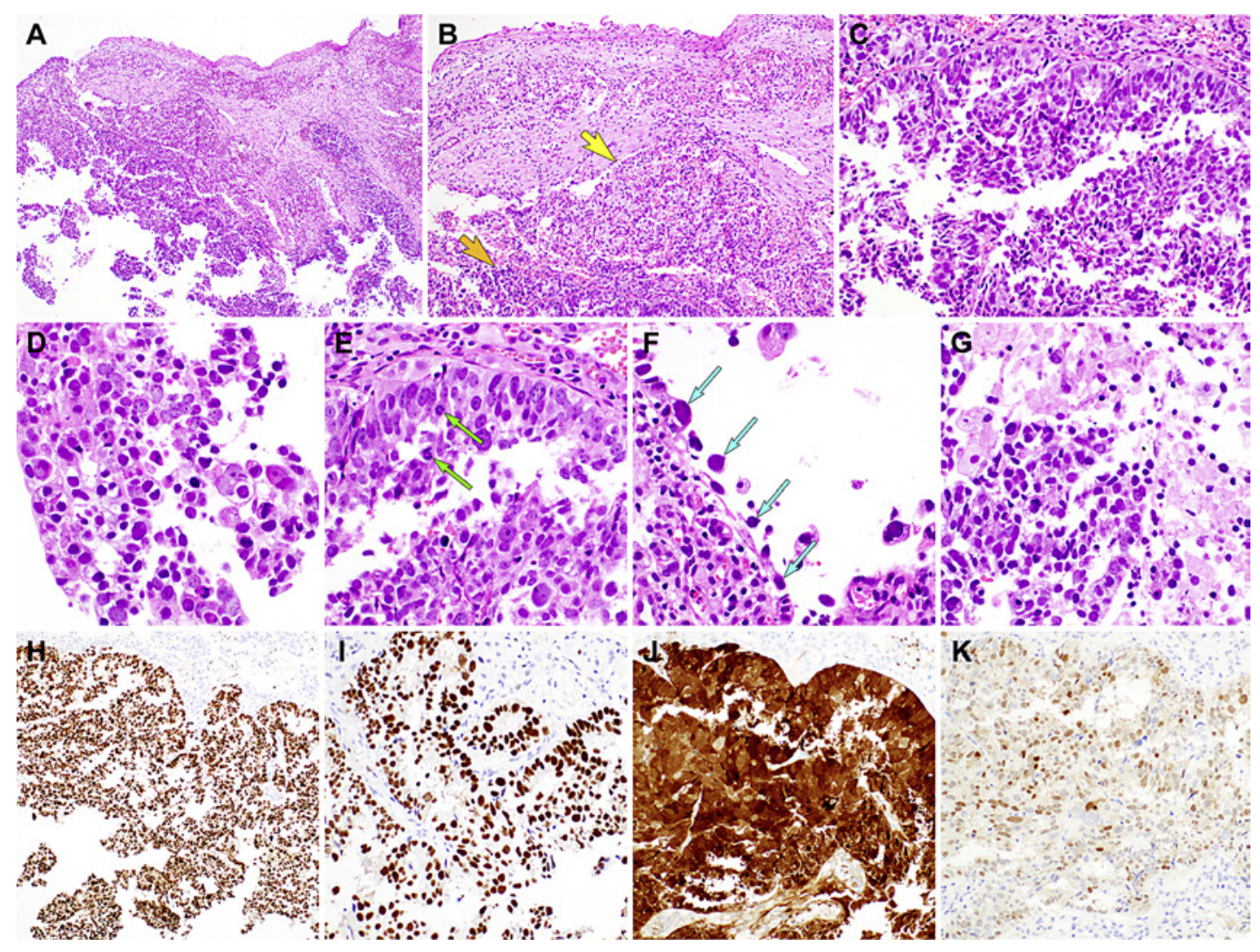

Fig. 2. Histological features and immunostaining results of the punch biopsy specimen collected from the vaginal stump. A The tumor tissue showed irregular infiltration of the subepithelial stroma. B In addition to the tumor cells (orange arrow), inflammatory cells, histiocytes, and red blood cells were noted (yellow arrow). C The tumor glands showed solid architecture and some small glandular lumina at the periphery. D High-power view revealed the tumor cells had enlarged, severely pleomorphic nuclei showing hyperchromasia and conspicuous nucleoli. E Atypical mitotic figures were occasionally identified. F Some tumor cell areas showed hobnail appearance. G There were a few microscopic foci of coagulative tumor cell necrosis. H, I p53 immunostaining revealed diffuse and strong nuclear expression, indicating missense TP53 mutation. J The tumor cells exhibited diffuse and strong nuclear and cytoplasmic p16 immunoreactivity, confirming the diagnosis of metastatic serous carcinoma from the endometrium. K Estrogen receptor expression was focal and weak. Staining method: hematoxylin and eosin staining (A-G); polymer method (H-K). Magnification: $\times 40$ (A); $\times 100$ (B); $\times 200$ (C); $\times 400$ (D-G); $\times 100$ (H); $\times 200(\mathbf{I}-\mathbf{K})$.

Despite the minimal volume of primary intrauterine tumor, the patient developed peritoneal metastases, which are extremely unusual for low-grade endometrioid carcinoma of which the tumor volume is minimal and myometrial invasion is absent. Histological features and immunostaining results observed in both hysterectomy and vaginal punch biopsy specimens are compatible with MUSC and its metastasis, respectively.

\section{Discussion}

Although MUSCs are confined to the endometrium proper, they have highly metastatic potential to disseminate to extra-uterine sites. Our patient was initially diagnosed with early-stage low-grade endometrioid carcinoma and accordingly underwent total hyster- 


\section{Case Reports in Oncology}

\begin{tabular}{l|l}
\hline Case Rep Oncol 2020;13:1537-1544 \\
\hline DOI: 10.1159/000511701 & $\begin{array}{l}\text { @ 2020 The Author(s). Published by S. Karger AG, Basel } \\
\text { www.karger.com/cro }\end{array}$ \\
\hline
\end{tabular}

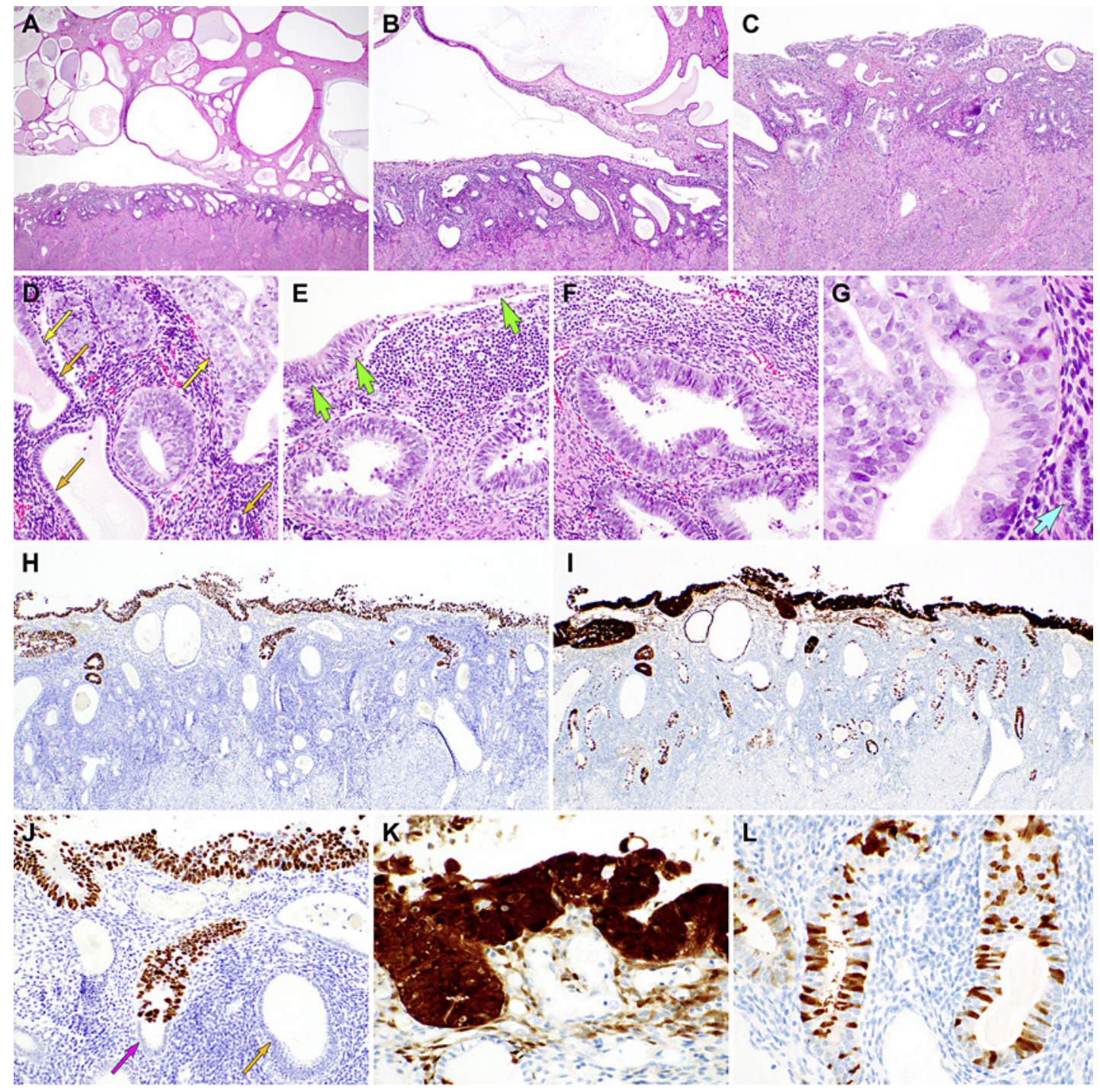

Fig. 3. Histological features and immunostaining results of the hysterectomy specimen obtained from the primary uterine tumor. A-B There is an endometrial polyp showing extensive cystic change and stromal fibrosis. C The non-polypoid endometrium adjacent to the endometrial polyp showed atypical glandular proliferation. D The tumor glands (yellow arrows) extended along the epithelial lining of atrophic endometrial glands. The uninvolved epithelium is indicated by orange arrows. E The surface epithelium (green arrows) also showed serous carcinoma (serous endometrial intraepithelial carcinoma). F Mixed inflammatory infiltrates and fibrosis were noted in the stroma. G High-power view revealed marked nuclear atypia of serous carcinoma compared to an atrophic endometrial gland (blue arrow). $\mathbf{H}, \mathbf{I}$ On immunostaining, the tumor cells exhibited diffuse and strong expression for p53 (H) and p16 (I). J The purple arrow indicates an endometrial gland partially involved by the tumor. The orange arrow indicates an atrophic endometrial gland that is free of the tumor. $\mathbf{K}$ p16 expression is uniform and strong in the nuclei and cytoplasm of the tumor cells. L Atrophic endometrial glands exhibited patchy p16 expression. Staining method: hematoxylin and eosin staining (A-G); polymer method (H-L). Magnification: $\times 12.5(\mathbf{A}, \mathbf{B}) ; \times 40$ (C); $\times 100(\mathbf{D}-\mathbf{F}) ; \times 200(\mathbf{G}) ; \times 40(\mathbf{H}$, I); $\times 100$ (J); $\times 200(\mathbf{K}, \mathbf{L})$. 
ectomy without postoperative adjuvant treatment. However, 7 years postoperatively, she developed a metastatic recurrence. To determine the cause of discrepancy between the clinical course and pathological diagnosis, we reviewed the hysterectomy slides and compared the histological features between the primary uterine and vaginal metastatic tumor. Both lesions showed characteristic morphologies of uterine serous carcinoma. We considered that the misinterpretation of histological type in the hysterectomy specimen might have been caused by the following: 1) the tumor volume was smaller than that of typical serous carcinoma; 2 ) the small separate tumor foci were confined within the endometrium proper, and there was no myometrial invasion; 3) the tumor consisted predominantly of well-formed glandular structures; and 4) high-grade nuclear atypia was initially overlooked.

The prognosis of uterine serous carcinoma is significantly associated with the pathological stage at presentation [5-8]. However, there have also been patients with stage I disease who developed fatal recurrences $[9,10]$. Because a few microscopic foci of metastatic carcinoma are usually the only evidence of intra-abdominal metastases, comprehensive surgical staging followed by an extensive sampling of the specimen and a careful histological examination is important for selecting the appropriate therapeutic strategy and predicting the prognosis of patients with serous carcinoma. In this study, the possibility of microscopic omental or peritoneal metastases at the time of hysterectomy cannot be completely ruled out because our patient did not undergo omentectomy or peritoneal biopsy.

A subset of uterine serous carcinoma that shows a prominent glandular pattern and superficial localization simulates low-grade endometrioid carcinoma. The hysterectomy specimen of our patient showed architecturally well-formed glands closely resembling those of low-grade endometrioid carcinoma at low-power magnification. Moreover, serous endometrial intraepithelial carcinoma replacing the surface epithelium can be easily overlooked or misinterpreted as reparative epithelium. However, cytologically, the tumor cells show high-grade nuclear atypia including high nuclear-to-cytoplasmic ratio, severe enlargement and pleomorphism, conspicuous nucleoli, and brisk mitotic activity. In uterine serous carcinoma, there is usually a significant discordance between architectural and cytological features, whereas obvious endometrioid differentiation (e.g., squamous, mucinous, or secretory change) is absent. In addition, we found that the tumor glands were randomly distributed in a background of atrophic endometrium. This finding does not support the diagnosis of low-grade endometrioid carcinoma, almost all of which develops from hyperplasia resulting from unopposed estrogenic stimulation.

The therapeutic benefit of adjuvant therapy in patients with MUSC is yet to be clarified [11-13]. However, additional treatment, re-staging, and short-term clinical follow-up may be necessary to correctly determine the extent of disease and improve the clinical outcomes in patients who underwent incomplete surgical staging.

MUSC has a propensity to involve benign endometrial polyps [14, 15]. In our case, there was no tumor involvement in an endometrial polyp; rather, all tumor foci were identified in non-polypoid endometrium adjacent to the endometrial polyp.

In summary, the case findings support that failure to preoperatively recognize high-risk endometrial carcinoma is associated with worse outcomes. We highlighted the critical and unique features of MUSC and discussed the potential for histological misinterpretation and the importance of recognizing MUSC. Accurate diagnosis and complete surgical staging are critical for patients with serous carcinoma even at a clinically early stage. The recognition of MUSC will provide the best opportunity to optimize the care of patients with this uncommon form of endometrial carcinoma.

\section{Karger 'ह}




\section{Case Reports in Oncology}

\begin{tabular}{l|l}
\hline Case Rep Oncol 2020;13:1537-1544 \\
\hline DOI: 10.1159/000511701 & $\begin{array}{l}\text { @ 2020 The Author(s). Published by S. Karger AG, Basel } \\
\text { www.karger.com/cro }\end{array}$ \\
\hline
\end{tabular}

Han/Kim: Minimal Uterine Serous Carcinoma

\section{Acknowledgment}

The authors would like to thank Dr. Sung-Im Do (Department of Pathology, Kangbuk Samsung Hospital, Sungkyunkwan University School of Medicine, Seoul, Korea) for her valuable comments and support.

\section{Statement of Ethics}

This study (2020-09-016) was reviewed and approved by the Institutional Review Board of Samsung Medical Center (Seoul, Korea). Written informed consent was obtained from the patient for publication of this case report and any accompanying images.

\section{Conflict of Interest Statement}

The authors have no conflicts of interest to declare.

\section{Funding Sources}

This research was supported by the National Research Foundation of Korea (NRF) grant funded by the Korean government (Ministry of Science and ICT) (2018R1C1B5043725).

\section{Author Contributions}

Jiheun Han: conceptualization, data collection, data analysis, manuscript drafting, and manuscript editing; Hyun-Soo Kim: conceptualization, data collection, data analysis, manuscript drafting, manuscript editing, funding acquisition, and supervision. All authors read and approved the final manuscript.

\section{References}

1 Baergen RN, Warren CD, Isacson C, Ellenson LH. Early uterine serous carcinoma: clonal origin of extrauterine disease. Int J Gynecol Pathol. 2001 Jul;20(3):214-9.

2 Ambros RA, Sherman ME, Zahn CM, Bitterman P, Kurman RJ. Endometrial intraepithelial carcinoma: a distinctive lesion specifically associated with tumors displaying serous differentiation. Hum Pathol. 1995 Nov; 26(11):1260-7.

3 Jia L, Yuan Z, Wang Y, Cragun JM, Kong B, Zheng W. Primary sources of pelvic serous cancer in patients with endometrial intraepithelial carcinoma. Mod Pathol. 2015 Jan;28(1):118-27.

4 Wheeler DT, Bell KA, Kurman RJ, Sherman ME. Minimal uterine serous carcinoma: diagnosis and clinicopathologic correlation. Am J Surg Pathol. 2000 Jun;24(6):797-806.

5 Craighead PS, Sait K, Stuart GC, Arthur K, Nation J, Duggan M, et al. Management of aggressive histologic variants of endometrial carcinoma at the Tom Baker Cancer Centre between 1984 and 1994. Gynecol Oncol. 2000 May; 77(2):248-53.

6 Benito V, Lubrano A, Arencibia O, Alvarez EE, León L, Medina N, et al. Pure papillary serous tumors of the endometrium: a clinicopathological analysis of 61 cases from a single institution. Int J Gynecol Cancer. 2009 Nov; 19(8):1364-9.

7 Slomovitz BM, Burke TW, Eifel PJ, Ramondetta LM, Silva EG, Jhingran A, et al. Uterine papillary serous carcinoma (UPSC): a single institution review of 129 cases. Gynecol Oncol. 2003 Dec;91(3):463-9.

8 Black C, Feng A, Bittinger S, Quinn M, Neesham D, McNally O. Uterine Papillary Serous Carcinoma: A SingleInstitution Review of 62 Cases. Int J Gynecol Cancer. 2016 Jan;26(1):133-40.

9 Gallion HH, van Nagell JR Jr., Powell DF, Donaldson ES, Higgins RV, Kryscio RJ, et al. Stage I serous papillary carcinoma of the endometrium. Cancer. 1989 Jun 1;63(11):2224-8.

\section{Karger'א}


10 Carcangiu ML, Tan LK, Chambers JT. Stage IA uterine serous carcinoma: a study of 13 cases. Am J Surg Pathol. 1997 Dec;21(12):1507-14.

11 Kelly MG, O'Malley DM, Hui P, McAlpine J, Yu H, Rutherford TJ, et al. Improved survival in surgical stage I patients with uterine papillary serous carcinoma (UPSC) treated with adjuvant platinum-based chemotherapy. Gynecol Oncol. 2005 Sep;98(3):353-9.

12 Velker V, D'Souza D, Prefontaine M, McGee J, Leung E. Role of Adjuvant Therapy for Stage IA Serous and Clear Cell Uterine Cancer: Is Observation a Valid Strategy? Int J Gynecol Cancer. 2016 Mar;26(3):491-6.

13 Thomas MB, Mariani A, Cliby WA, Keeney GA, Podratz KC, Dowdy SC. Role of systematic lymphadenectomy and adjuvant therapy in stage I uterine papillary serous carcinoma. Gynecol Oncol. 2007 Nov;107(2):186-9.

14 Silva EG, Jenkins R. Serous carcinoma in endometrial polyps. Mod Pathol. 1990 Mar;3(2):120-8.

15 Trahan S, Têtu B, Raymond PE. Serous papillary carcinoma of the endometrium arising from endometrial polyps: a clinical, histological, and immunohistochemical study of 13 cases. Hum Pathol. 2005 Dec;36(12): 1316-21. 